\title{
Group Acceptance Sampling Plans for Resubmitted lots for life tests based on Half Logistic Distribution
}

\author{
G.Srinivasa $\mathrm{Rao}^{1}$ and B.Srinivasa $\mathrm{Rao}^{2 *}$ \\ ${ }^{1}$ Department of Statistics, The University of Dodoma \\ ${ }^{2}$ Department of Mathematics \& Humanities, R.V.R \& J.C College of Engineering
}

\begin{abstract}
In this article, a group acceptance sampling plan (GASP) for lot resubmitting is developed to ensure quality of the product lifetime assuming that the product's lifetime follows the half logistic distribution. The parameters of the GASP are determined by satifying the specified producer's and consumer's risks according to the experiment termination time and the number of testers. A comparison between this proposed group sampling and the ordinary group sampling plan is discussed. This proposed plan is justified with an illustration.
\end{abstract}

Key words: resubmitted lot, group sampling plan, life test, producer's risk, cosumer's risk

\section{Introduction}

The single acceptance sampling is the most popularly used one because it is simple for practical implementation. The decision on the lot acceptance/ rejection by the single acceptance sampling is based on the single inspection or life test. Today, products are produced to be of high reliability. To collect product lifetime information, products must suffer a destructive life test. Due to the fact that it could take long experimental time to observe the complete lifetime of a high reliability item, the life test must be ended within a specified schedule and such life test is called a truncated life test. Single acceptance sampling plans under a truncated life test have been investigated in the past few decades by Gupta and Groll (1961) [13], Gupta (1962) [14], Govindaraju and Ganesalingam (1997) [12], Fertig and Mann (1980) [11], Kantam and Rosaiah (1998) [15], Kantam et al. (2001) [16], Baklizi (2003) [7], Wu and Tsai (2005) [33], Rosaiah and Kantam (2005) [20], Tsai and Wu (2006) [32], Balakrishnan et al. (2007) [9], Aslam (2007) [1], Rao et al. (2008) [22], Aslam and Kantam (2008) [2], Rao et al. (2009) [23], Lio et al. (2010) [18], Rao et al. (2010) [25], Rao (2011) [26].

To save time and cost in the life test, it is often to put a number of items in a tester. In this life test, a tester is called a group and the number of items in each tester is called the group size. The acceptance sampling via the group life test is called the group acceptance sampling plan (GASP) which is also often implemented under a truncated life test. GASP based on different

\footnotetext{
${ }^{*}$ Corresponding author.
} 
distributions have been studied in the recent past by Aslam et al. (2009) [3], Aslam et al. (2009a) [4], Rao (2009) [24], Aslam et al. (2011) [5], Aslam et al. (2011a) [6], Ramaswamy and Anburajan (2012) [19].

In the single acceptance-sampling plan, the decision about rejecting or accepting the production lot is based on a single sample selected from the lot. In some cases, a production lot is not accepted by the consumer based on a single acceptance sampling plan, the producer may dispute the first sample results, take the second sample of the same size for testing, and make a decision discarding the first sample results based on the provisions of the contract or statutes. Hence, the rejected lot may be resubmitted. This repeated sampling process is called resubmitted sampling plan.

If a lot is not accepted on the first inspection by a single sampling plan, the resubmitted sampling plan provides the opportunity for the producer to test/recheck the rejected lot/product and then resubmit the rejected lot/product. Often, the producer has empirical information about the product and may like this re-sampling process. The GASP under the truncated life test could be applied to the resubmitted production lots. However, the GASP under the truncated life test for the resubmitted lot has not been discussed in the literature. The objective of this article is to use the concept of the repeated sampling plan for the resubmitted lots to develop the GASP under the truncated life test assuming that the product lifetimes have half logistic distribution. The rest of this article is organized as follows. In Section 2, the design of group acceptance sampling for lifetime percentiles under a truncated life test is described for the resubmitted lot. In Section 3, the proposed group sampling plan for the resubmitted lot is discussed with an illustration. Finally, conclusions are given in Section 4.

\section{Design of Group Acceptance Sampling for resubmitted lots}

The assumptions of the sampling inspection for the resubmitted lots are as follows:

(1) Abiding by the provisions of a contract or statute, the information of the original inspection resulting in non-acceptance is required to discard the lot.

(2) Producer is honest and consumer has confidence in producer who will not deliberately take the advantage of re-sampling.

The operating procedure of the proposed GASP under a truncated life test for resubmitted lots is implemented as follows:

Step1: Perform the original group sampling inspection by randomly selecting $n$ items and distributing them into $g$ groups evenly such that each group has $r$ items and $n=r g$. Decide about the experiment time period, $t_{0}$, and the acceptance number $c$. Accept the lot if the number of failures from all $g$ groups is not larger than $c$ within the $t_{0}$; otherwise, the lot is not accepted. Truncate the experiment once the number of failures from all the groups exceeds $c$, or the time of the experiment is ended, which occurs first.

Step2: On non-acceptance of lot from Step-1, apply the referenced group sampling plan at most $w$ times and reject the lot on the $w^{t h}$ inspection if 
the lot could not be accepted before or at the resubmission. Here the referenced group sampling plan is the original group sampling plan since resubmission is allowed.

It is important to notice that the proposed repeated group sampling plan based on the number of failures is not a simple extension of the sampling plan for the resubmitted lots.

Therefore, the proposed group sampling plan of the resubmitted lot cannot be compared with the single sampling plan of the resubmitted lot. Although the double group sampling and the multiple group sampling based on a truncated life test could be designed for the resubmitted lots, however, practically the ordinary (or single) group sampling under a truncated life test is preferred over the double group sampling and the multiple group sampling schemes due to its easiness of implementation. The Half logistic distribution, received more attention by the researchers due to its broad applications in different fields including the areas of reliability, failure time modeling, and acceptance-sampling plan. Balakrishnan seems to be the person who introduced this distribution in the area of quality control. The applications of half logistic distribution in various fields can be seen in Balakrishnan (1985) [8], Rosaiah et al. (2009) [21], Rao and Kantam (2011) [27], Rao and Kantam (2011a) [28], Rao and Kantam (2012) [29], Rao and Kantam (2012a) [30]. The probability density function (pdf) of a half logistic distribution is given by

$$
p(x)=\frac{2 e^{-x}}{\left(1+e^{-x}\right)}, \quad x \geq 0
$$

Its cumulative distribution function (cdf) is

$$
P(x)=\frac{\left(1-e^{-x}\right)}{\left(1+e^{-x}\right)}, \quad x \geq 0
$$

Half logistic distribution is an increasing failure rate (IFR) model which is most useful in reliability studies. Because of this IFR nature we are motivated to study this distribution. Assume that the life time of a product follows half logistic distribution with $\sigma$ as scale parameter. Its cumulative distribution function $\mathrm{F}($.$) is given by$

$$
F(t)=\frac{1-e^{-\frac{t}{\sigma}}}{1+e^{-\frac{t}{\sigma}}}, \quad t \geq 0, \sigma>0
$$

Given $0<\mathrm{q}<1$, the $100 \mathrm{q}^{\text {th }}$ percentile is given by

$$
t_{q}=\operatorname{alog}\left(\frac{1+q}{1-q}\right)
$$

Substituting $\sigma$ in the Equation 2.0.1 in the scaled form we get 


$$
\begin{gathered}
F(t)=\frac{1-e^{-\left(\frac{t}{t_{q}}\right) \log \left(\frac{1+q}{1-q}\right)}}{1+e^{-\left(\frac{t}{t q}\right) \log \left(\frac{1+q}{1-q}\right)}} \\
F(t)=\frac{1-e^{-\delta \log \left(\frac{1+q}{1-q}\right)}}{1+e^{-\delta \log \left(\frac{1+q}{1-q}\right)}}
\end{gathered}
$$

where $\delta=\frac{t}{t_{q}}$. In particular if we take $\mathrm{q}=0.50$, since for our present skewed population the median is a more approximate average for decision making about the quality of the life than population mean. Thus we may conclude that population median based sampling plan of half logistic distribution model is more economical than those based on population mean with respect to sample size. Let $\mu$ be the true value of the median of the lifetime distribution of a product and $\mu_{0}$ denote the specified median under the assumption that the life time of an item follows half logistic distribution. Based on the failure data, we want to test the hypothesis $H_{0}: \mu \geq \mu_{0}$ against $H_{1}: \mu<\mu_{0}$ A lot is considered as good if $\mu \geq \mu_{0}$ and bad if $\mu<\mu_{0}$. This hypothesis is tested using the group acceptance sampling scheme as:

1. Select the number of groups $g$ and allocate predefined $r$ items to each group so that the sample size for a lot will be $n=g . r$.

2. Select the acceptance number $\mathrm{c}$ for a group and the experiment time $t_{0}$.

3. Perform the experiment for the g groups simultaneously and record the number of failures for each group.

4. Accept the lot if at most $\mathrm{c}$ failures occur in each of all groups.

5. Terminate the experiment if more than $\mathrm{c}$ failures occur in any group and reject the lot.

We are interested in determining the number of groups g required in the case of the half logistic distribution and various values of acceptance number $c$, whereas the group size $r$ and the termination time $t_{0}$ are assumed to be given. Since it is convenient to set the termination time as a multiple of the specified value $\mu_{0}$ of the median, we will consider $t_{0}=\delta \mu_{0}$ for a given constant $\delta$ (termination ratio). The probability $(\alpha)$ of rejecting a good lot is called the producer's risk, whereas the probability $(\beta)$ of accepting a bad lot is known as the consumer's risk. The parameter value $g$ of the proposed sampling plan is determined for ensuring the consumer's risk $\beta$. Often, the consumer's risk $\beta$ is expressed by the consumer's confidence level. If the confidence level is $p^{*}$, then the consumer's risk will be $\beta=1-p^{*}$. We will determine the number of groups $\mathrm{g}$ in the proposed sampling plan so that the consumer's risk does not exceed a given value $\beta$. If the lot size is large enough, we can use the binomial distribution to develop the GASP. According to the GASP the lot of products is accepted only if there are at most $\mathrm{c}$ failures observed in each of the g groups. So, the lot acceptance probability as in Aslam et al. (2009a) [4] is given by

$$
\mathrm{L}(\mathrm{p})=\sum_{i=0}^{c}\left(\begin{array}{c}
\mathrm{rg} \\
\mathrm{i}
\end{array}\right) \mathrm{p}^{\mathrm{i}}(1-\mathrm{p})^{\mathrm{rg}-\mathrm{i}}
$$


According to Aslam et al. (2011a) [6] the OC functon of sampling plan for the resubmitted lots with $w-1$ resubmissions is given by

$$
\mathrm{P}_{\mathrm{a}}(\mathrm{p})=1-(1-\mathrm{L}(\mathrm{p}))^{w}
$$

Therefore the OC function of the proposed GASP can be given by

$$
\mathrm{P}_{\mathrm{a}}(\mathrm{p})=1-\left(1-\sum_{i=0}^{c}\left(\begin{array}{c}
\mathrm{rg} \\
\mathrm{i}
\end{array}\right) \mathrm{p}^{\mathrm{i}}(1-\mathrm{p})^{\mathrm{rg}-\mathrm{i}}\right)^{w}
$$

There are two parameters, $c$ and $g$, in this proposed GASP for the resubmitted lot, given the group size $r$ and the pre-specified truncated life test time schedule, $t_{0}=\delta_{q} t_{q}^{0}$, in terms of a multiple of targeted lifetime percentile, $t_{q}^{0}$ is greater than or equal to the target one, the product is called a good product lot; otherwise it is called a bad lot. Usually, there are two risks attached with an acceptance sampling plan. The chance of rejecting a good lot is called the producer's risk $\alpha$ and the chance of accepting a bad lot is called consumer's risk $\beta$. Both producer and consumer want a sampling plan to make the decision satisfy their respective specified risks. Producer requires the lot acceptance probability at least $1-\alpha$ at the acceptability reliability level (ARL) and consumers want a sampling plan to make the decision satisfy their respective specified risks. Producer requires the lot acceptance probability at least 1- $\alpha$ at the acceptable reliability level (ARL) and the consumer wants the lot acceptance probability less than $\beta$ at the lot tolerance reliability level (LTRL). Let $p_{1}$ be probability of a failure corresponding to the producer's risk at ARLs, in terms of $\frac{t_{q}}{t_{q}^{0}}=2,4,6,8,10$. And let $p_{2}$ be the probability of a failure corresponding to the consumer's risk at LTRL, in terms of $\frac{t_{q}}{t_{q}^{0}}=1$. Therefore the plan parameters $c$ and $g$ can be determined by solving the following inequalities simultaneously,

$$
1-\left(1-\sum_{i=0}^{c}\left(\begin{array}{c}
r g \\
i
\end{array}\right) p_{1}^{i}\left(1-p_{1}\right)^{r g-i}\right)^{w} \geq 1-\alpha
$$

and

$$
1-\left(1-\sum_{i=0}^{c}\left(\begin{array}{c}
r g \\
i
\end{array}\right) p_{2}^{i}\left(1-p_{2}\right)^{r g-i}\right)^{w} \leq \beta
$$

Given the producer's risk $\alpha=0.05$, termination time schedule $t_{0}=\delta_{q} t_{q}^{0}$ and $t_{q}^{0}=0.5$ and 1.0, the two parameters of the proposed GASP under the truncated life test at the pre-specified time $t_{0}$, for the resubmitted lot with $\mathrm{w}=2,3$ are obtained according to the consumer's confidence levels $P^{*}=1-\beta=0.75,0.90,0.95$ and 0.99 , for $\mathrm{w}=2$ and the sampling parameters are shown in Table 1 (for $10^{\text {th }}$ percentile lifetime) and Table 2 (for median life time). For given $\beta, \frac{t_{0}}{t_{q}^{0}}$ and $\mathrm{r}$ at the same respective levels. Table 1 indicates that the number of groups $(\mathrm{g})$ needed for the proposed sampling plan is decreasing and acceptance number $c$ is equal or decreasing as $\delta_{q}$ increases from 
0.5 to 1 . However, Table 2 shows that both $g$ and $c$ are increasing as $\delta_{q}$ increases from 0.5 to 1.0 . For $\mathrm{w}=3$, the sampling parameters are displayed in Tables 3 (for $10^{\text {th }}$ percentile lifetime) and Table 4 (for median lifetime). Table 3 indicates that the number of groups ( $\mathrm{g}$ ) needed for the proposed sampling plan is decreasing $\frac{t_{0}}{t_{q}^{0}}=2$ and the acceptance number $c$ is increasing as $\delta_{q}$ increases from 0.5 to 1.0. Whereas, Table 4 shows that both $g$ and $c$ are increasing from 0.5 to 1.0 .

Table 1: Proposed plan having $w=2$ under half logistic distribution for $10^{\text {th }}$ percentile

\begin{tabular}{|c|c|c|c|c|c|c|c|c|c|c|c|c|c|}
\hline & & \multicolumn{9}{|c|}{$\mathrm{r}=5$} & \multicolumn{6}{c|}{$\mathrm{r}=10$} \\
\hline & & \multicolumn{3}{|c|}{$\delta_{q}=0.5$} & \multicolumn{3}{|c|}{$\delta_{q}=1.0$} & \multicolumn{3}{|c|}{$\delta_{q}=0.5$} & \multicolumn{3}{|c|}{$\delta_{q}=1.0$} \\
\hline$\beta$ & $\frac{t_{q}}{t_{q}^{0}}$ & $\mathrm{c}$ & $\mathrm{g}$ & $P_{a}$ & $\mathrm{c}$ & $\mathrm{g}$ & $P_{a}$ & $\mathrm{c}$ & $\mathrm{g}$ & $P_{a}$ & $\mathrm{c}$ & $\mathrm{g}$ & $P_{a}$ \\
\hline 0.25 & 2 & 7 & 45 & 0.9575 & 7 & 22 & 0.9649 & 7 & 23 & 0.9504 & 7 & 11 & 0.9649 \\
& 4 & 1 & 14 & 0.9520 & 1 & 7 & 0.9521 & 1 & 7 & 0.9520 & 2 & 5 & 0.9830 \\
& 6 & 1 & 14 & 0.9864 & 1 & 7 & 0.9866 & 1 & 7 & 0.9864 & 1 & 4 & 0.9792 \\
& 8 & 0 & 8 & 0.9505 & 0 & 4 & 0.9502 & 0 & 4 & 0.9505 & 0 & 2 & 0.9502 \\
\hline 0.1 & 2 & 11 & 72 & 0.9611 & 11 & 36 & 0.9623 & 11 & 36 & 0.9611 & 11 & 18 & 0.9623 \\
& 4 & 2 & 25 & 0.9569 & 2 & 13 & 0.9502 & 3 & 16 & 0.9796 & 3 & 8 & 0.9799 \\
& 6 & 1 & 19 & 0.9643 & 1 & 10 & 0.9585 & 1 & 10 & 0.9584 & 1 & 5 & 0.9585 \\
& 8 & 1 & 19 & 0.9856 & 1 & 10 & 0.9831 & 1 & 10 & 0.9830 & 1 & 5 & 0.9831 \\
\hline 0.05 & 2 & 13 & 88 & 0.9518 & 13 & 44 & 0.9531 & - & - & - & 13 & 22 & 0.9531 \\
& 4 & 3 & 35 & 0.9681 & 3 & 17 & 0.9727 & 3 & 18 & 0.9635 & 3 & 9 & 0.9639 \\
& 6 & 2 & 29 & 0.9850 & 2 & 14 & 0.9873 & 2 & 15 & 0.9826 & 2 & 7 & 0.9873 \\
& 8 & 1 & 22 & 0.9769 & 1 & 11 & 0.9770 & 1 & 11 & 0.9952 & 1 & 6 & 0.9698 \\
\hline 0.01 & 2 & 20 & 137 & 0.9582 & 19 & 66 & 0.9505 & 20 & 69 & 0.9544 & 19 & 33 & 0.9505 \\
& 4 & 4 & 50 & 0.9571 & 4 & 25 & 0.9576 & 20 & 69 & 1.0000 & 5 & 14 & 0.9800 \\
& 6 & 2 & 37 & 0.9590 & 2 & 18 & 0.9634 & 20 & 69 & 1.0000 & 2 & 9 & 0.9634 \\
& 8 & 2 & 37 & 0.9876 & 2 & 18 & 0.9891 & 20 & 69 & 1.0000 & 2 & 9 & 0.9891 \\
\hline
\end{tabular}


Table 2: Proposed plan having $w=2$ under half logistic distribution for $50^{\text {th }}$ percentile

\begin{tabular}{|c|c|c|c|c|c|c|c|c|c|c|c|c|c|}
\hline & & \multicolumn{9}{c|}{$\mathrm{r}=5$} & \multicolumn{6}{c|}{$\mathrm{r}=10$} \\
\hline & & \multicolumn{3}{|c|}{$\delta_{q}=0.5$} & \multicolumn{2}{|c|}{$\delta_{q}=1.0$} & \multicolumn{3}{|c|}{$\delta_{q}=0.5$} & \multicolumn{3}{|c|}{$\delta_{q}=1.0$} \\
\hline$\beta$ & $\frac{t_{q}}{t_{q}}$ & $\mathrm{c}$ & $\mathrm{g}$ & $P_{a}$ & $\mathrm{c}$ & $\mathrm{g}$ & $P_{a}$ & $\mathrm{c}$ & $\mathrm{g}$ & $P_{a}$ & $\mathrm{c}$ & $\mathrm{g}$ & $P_{a}$ \\
\hline 0.25 & 2 & 7 & 8 & 0.9707 & - & - & - & - & - & - & - & - & - \\
& 4 & 2 & 4 & 0.9763 & 5 & 6 & 0.9523 & 6 & 7 & 0.9588 & - & - & - \\
& 6 & 1 & 3 & 0.9780 & 2 & 3 & 0.9770 & 2 & 3 & 0.9756 & 6 & 7 & 0.9588 \\
& 8 & 1 & 3 & 0.9915 & 1 & 2 & 0.9785 & 1 & 2 & 0.9778 & 6 & 7 & 0.9588 \\
\hline 0.10 & 2 & 9 & 11 & 0.9554 & - & - & - & - & - & - & - & - & - \\
& 4 & 3 & 6 & 0.9784 & 5 & 6 & 0.9523 & 6 & 7 & 0.9588 & - & - & - \\
& 6 & 2 & 4 & 0.9891 & 2 & 3 & 0.9770 & 2 & 3 & 0.9756 & 6 & 7 & 0.9588 \\
& 8 & 1 & 4 & 0.9778 & 1 & 2 & 0.9785 & 1 & 2 & 0.9778 & 6 & 7 & 0.9588 \\
\hline 0.05 & 2 & 12 & 15 & 0.9525 & - & - & - & - & - & - & - & - & - \\
& 4 & 3 & 6 & 0.9784 & 5 & 6 & 0.9523 & 6 & 7 & 0.9588 & - & - & - \\
& 6 & 2 & 5 & 0.9891 & 2 & 3 & 0.9770 & 2 & 3 & 0.9756 & 6 & 7 & 0.9588 \\
& 8 & 1 & 4 & 0.9778 & 1 & 2 & 0.9785 & 1 & 2 & 0.9778 & 6 & 7 & 0.9588 \\
\hline 0.01 & 2 & 18 & 23 & 0.9521 & - & - & - & - & - & - & - & - & - \\
& 4 & 4 & 9 & 0.9626 & 5 & 6 & 0.9523 & 6 & 7 & 0.9588 & - & - & - \\
& 6 & 2 & 7 & 0.9540 & 2 & 3 & 0.9770 & 3 & 4 & 0.9881 & 6 & 7 & 0.9588 \\
& 8 & 1 & 5 & 0.9554 & 2 & 3 & 0.9938 & 2 & 4 & 0.9753 & 6 & 7 & 0.9588 \\
\hline
\end{tabular}

Table 3: Proposed plan having $w=3$ under half logistic distribution for $10^{\text {th }}$ percentile

\begin{tabular}{|c|c|c|c|c|c|c|c|c|c|c|c|c|c|}
\hline & & \multicolumn{9}{|c|}{$\mathrm{r}=5$} & \multicolumn{6}{c|}{$\mathrm{r}=10$} \\
\hline & & \multicolumn{3}{|c|}{$\delta_{q}=0.5$} & \multicolumn{2}{|c|}{$\delta_{q}=1.0$} & \multicolumn{3}{|c|}{$\delta_{q}=0.5$} & \multicolumn{3}{|c|}{$\delta_{q}=1.0$} \\
\hline$\beta$ & $\frac{t_{q}}{t_{q}^{0}}$ & $\mathrm{c}$ & $\mathrm{g}$ & $P_{a}$ & $\mathrm{c}$ & $\mathrm{g}$ & $P_{a}$ & $\mathrm{c}$ & $\mathrm{g}$ & $P_{a}$ & $\mathrm{c}$ & $\mathrm{g}$ & $P_{a}$ \\
\hline 0.25 & 2 & 5 & 38 & 0.9599 & 5 & 19 & 0.9603 & 5 & 19 & 0.9599 & 6 & 11 & 0.9690 \\
& 4 & 1 & 16 & 0.9813 & 5 & 19 & 1.0000 & 1 & 8 & 0.9813 & 1 & 4 & 0.9813 \\
& 6 & 0 & 10 & 0.9597 & 5 & 19 & 1.0000 & 0 & 5 & 0.9597 & 1 & 4 & 0.9970 \\
& 8 & 0 & 10 & 0.9803 & 5 & 19 & 1.0000 & 0 & 5 & 0.9803 & 0 & 3 & 0.9687 \\
\hline 0.10 & 2 & 8 & 60 & 0.9605 & 7 & 27 & 0.9511 & 8 & 30 & 0.9605 & 8 & 15 & 0.9611 \\
& 4 & 2 & 27 & 0.9861 & 7 & 27 & 1.0000 & 2 & 14 & 0.9830 & 1 & 5 & 0.9541 \\
& 6 & 1 & 21 & 0.9895 & 7 & 27 & 1.0000 & 1 & 11 & 0.9871 & 1 & 5 & 0.9915 \\
& 8 & 0 & 14 & 0.9548 & 0 & 7 & 0.9545 & 0 & 7 & 0.9548 & 1 & 5 & 0.9978 \\
\hline 0.05 & 2 & 10 & 76 & 0.9543 & 10 & 38 & 0.9549 & 10 & 38 & 0.9543 & 10 & 19 & 0.9549 \\
& 4 & 2 & 31 & 0.9708 & 2 & 15 & 0.9754 & 2 & 16 & 0.9656 & 2 & 8 & 0.9657 \\
& 6 & 1 & 24 & 0.9813 & 1 & 12 & 0.9813 & 1 & 12 & 0.9813 & 1 & 6 & 0.9813 \\
& 8 & 1 & 24 & 0.9947 & 1 & 12 & 0.9948 & 1 & 12 & 0.9947 & 1 & 6 & 0.9948 \\
\hline 0.01 & 2 & - & - & - & 15 & 57 & 0.9544 & 16 & 60 & 0.9610 & 16 & 30 & 0.9617 \\
& 4 & 3 & 46 & 0.9651 & 3 & 23 & 0.9652 & 3 & 23 & 0.9651 & 3 & 12 & 0.9553 \\
& 6 & 2 & 39 & 0.9887 & 2 & 19 & 0.9904 & 2 & 20 & 0.9870 & 2 & 10 & 0.9871 \\
& 8 & 1 & 31 & 0.9836 & 1 & 16 & 0.9813 & 1 & 16 & 0.9813 & 1 & 8 & 0.9813 \\
\hline
\end{tabular}


Table 4: Proposed plan having $w=3$ under half logistic distribution for $50^{\text {th }}$ percentile

\begin{tabular}{|c|c|c|c|c|c|c|c|c|c|c|c|c|c|}
\hline & & \multicolumn{10}{c|}{$\mathrm{r}=5$} & \multicolumn{6}{c|}{$\mathrm{r}=10$} \\
\hline & & \multicolumn{3}{|c|}{$\delta_{q}=0.5$} & \multicolumn{3}{|c|}{$\delta_{q}=1.0$} & \multicolumn{3}{c|}{$\delta_{q}=0.5$} & \multicolumn{3}{|c|}{$\delta_{q}=1.0$} \\
\hline$\beta$ & $t_{q}$ & $\mathrm{c}$ & $\mathrm{g}$ & $P_{a}$ & $\mathrm{c}$ & $\mathrm{g}$ & $P_{a}$ & $\mathrm{c}$ & $\mathrm{g}$ & $P_{a}$ & $\mathrm{c}$ & $\mathrm{g}$ & $P_{a}$ \\
\hline 0.25 & $t_{q}^{0}$ & 5 & 7 & 0.9601 & - & - & - & - & - & - & - & - & - \\
& 4 & 1 & 3 & 0.9792 & 2 & 3 & 0.9619 & 2 & 3 & 0.9610 & - & - & - \\
& 6 & 1 & 3 & 0.9967 & 1 & 2 & 0.9878 & 1 & 2 & 0.9875 & 20 & 21 & 0.9517 \\
& 8 & 0 & 2 & 0.9744 & 0 & 1 & 0.9733 & 0 & 1 & 0.9744 & 2 & 3 & 0.9610 \\
\hline 0.10 & 2 & 8 & 11 & 0.9629 & - & - & - & - & - & - & - & - & - \\
& 4 & 2 & 5 & 0.9855 & 2 & 3 & 0.9619 & 2 & 3 & 0.9610 & - & - & - \\
& 6 & 1 & 4 & 0.9875 & 1 & 2 & 0.9878 & 1 & 2 & 0.9875 & 20 & 21 & 0.9517 \\
& 8 & 1 & 4 & 0.9967 & 0 & 1 & 0.9733 & 1 & 2 & 0.9967 & 2 & 3 & 0.9610 \\
\hline 0.05 & 2 & 10 & 14 & 0.9546 & - & - & - & - & - & - & - & - & - \\
& 4 & 2 & 6 & 0.9610 & 2 & 3 & 0.9619 & 2 & 3 & 0.9610 & - & - & - \\
& 6 & 1 & 4 & 0.9875 & 1 & 2 & 0.9878 & 1 & 2 & 0.9875 & 20 & 21 & 0.9517 \\
& 8 & 1 & 4 & 0.9967 & 1 & 2 & 0.9968 & 1 & 2 & 0.9967 & 2 & 3 & 0.9610 \\
\hline 0.01 & 2 & 15 & 21 & 0.9540 & - & - & - & - & - & - & - & - & - \\
& 4 & 3 & 8 & 0.9748 & 3 & 4 & 0.9763 & 3 & 4 & 0.9748 & - & - & - \\
& 6 & 2 & 7 & 0.9901 & 2 & 4 & 0.9795 & 2 & 4 & 0.9789 & 20 & 21 & 0.9517 \\
& 8 & 1 & 6 & 0.9791 & 1 & 3 & 0.9792 & 1 & 3 & 0.9791 & 2 & 3 & 0.9610 \\
\hline
\end{tabular}

Note: The cells with hyphens(-) indicate that $\mathrm{g}$ and $\mathrm{c}$ cannot be found to satisfy the conditions

\section{Illustrative Example}

In this section, an example with real data set is given to illustrate the proposed acceptance sampling plans. The data set is of the tests on endurance of deep groove ball bearings (Lawless (1982), p.228) [17]. The data are the number of million revolutions before failure for each of the 23 ball bearings in life test and they are: 17.88, 28.92, 33.00, 41.52, 42.12, 45.60, 48.80, 51.84, $51.96,54.12,55.56,67.80,68.44,68.64,68.88,84.12$, 93.12, 98.64, 105.12, 105.84, 127.92, 128.04 and 173.40 . As the confidence level is assured by this acceptance sampling plan only if the lifetimes are from the half logistic distribution, we should check if it is reasonable to admit that the given sample comes from the half logistic distribution by the goodness of fit test and model selection criteria. The data set was used by Sultan (2007) [31] to demonstrate the goodness of fit for generalized exponential distribution. However, the acceptance sampling plans under the truncated life test based on the half logistic distribution for percentiles have not yet been developed. We have applied R-square method, K-S test and QQ-plot to test the goodness of fit for the data set and we got R-square $=0.98205, \mathrm{~K}-\mathrm{S}$ value $=0.8696$ with $\mathrm{p}$-value $=0.3333$ and also the QQ-plot is given below. Therefore, it is clear that half logistic model fits quite well to the data. 


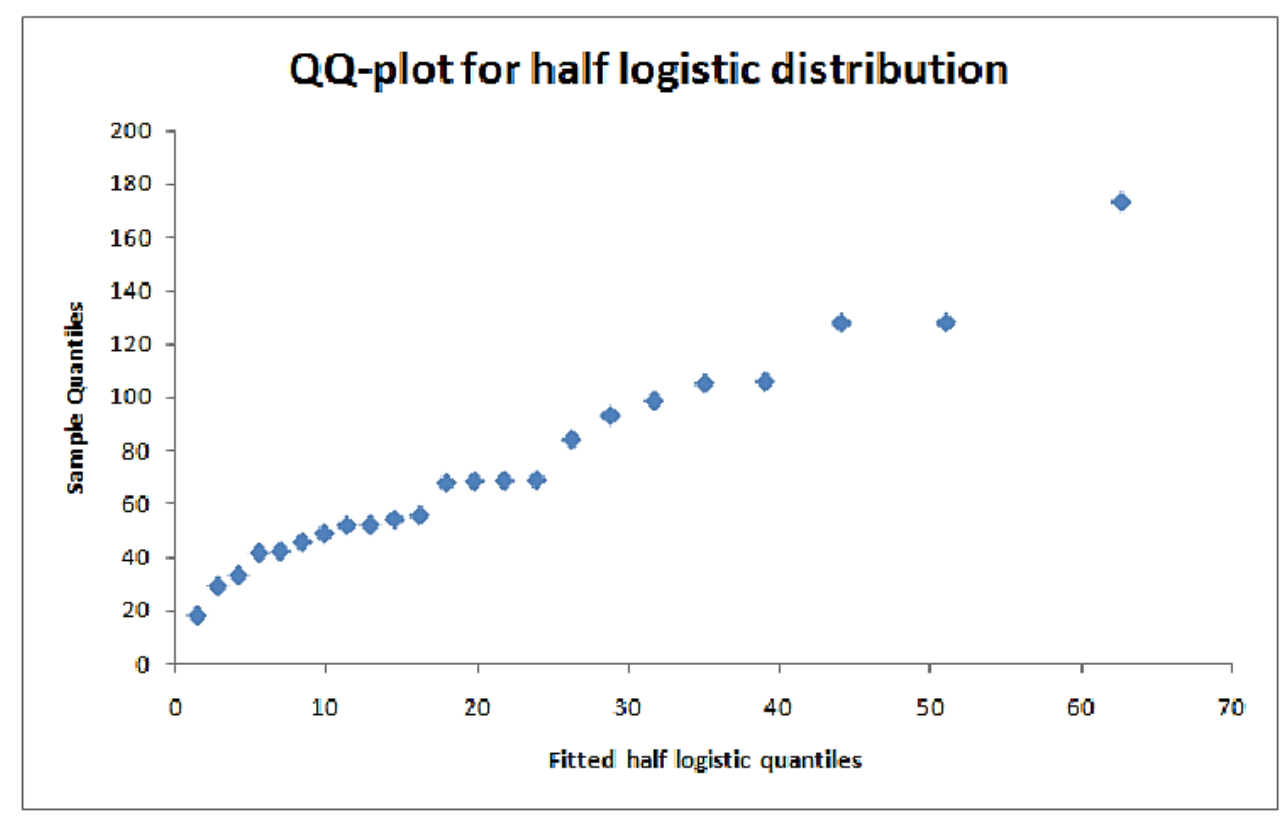

Suppose that an experimenter would like to establish the true unknown $50^{\text {th }}$ percentile life time for the number of million revolutions before the failure time at 40 million revolutions and protect the producer's risk with $\alpha=0.05$ at the level of 4 times of the specified median life. The experimenter would like to perform the life test using the proposed group sampling plan having $\mathrm{r}=5$ with resubmitted lot with $\mathrm{w}=2$. Let the termination ratio be $\delta_{q}=0.5$ and $\beta=0.25$, from table 2 , we can observe that $\mathrm{c}=2, \mathrm{~g}=4$ and the acceptance probability is 0.9763 . According to this plan if the number of million revolutions is larger than 2 from 4 groups, the lot could not be accepted at the fist inspection. And second inspection must be conducted again. The lot is accepted if the number of failures is less than or equal to 2 from 4 groups after 40 million number of revolutions. According to this plan, life test has been accepted because there are only 3 failures before the termination of 40 million number of revolutions. According to this plan, life test has been accepted because there are only 3 failures before the termination of 40 million number of revolutions.

\section{Summary and Conclusions}

In this paper, a group sampling plan to ensure the specified product lifetime percentile for a resubmitted lot has been developed for the half logistic distribution. The parameters $\mathrm{c}$ and $\mathrm{g}$ of the proposed sampling plan are determined by the so-called two-point method. In other words, these parameters are determined such that the lot acceptance probability is larger than $1-\alpha$ at the producer-specified quality level but the lot acceptance probability is smaller than $\beta$ at the consumer-specified quality level. Extensive tables have been provided for the industrial use according to various parameters and percentile values. It was observed that the number of groups 
required increases as the consumer's confidence increases, true quality decreases, or the number of resubmission decreases. More importantly, the sample size can be significantly reduced by allowing just a small number of resubmissions in the proposed sampling plan.

\section{Acknowledgments}

The authors thank the editor and the reviewers for their helpful suggestions, comments and encouragement, which helped in improving the final version of the paper.

\section{References}

[1] Aslam.M.(2007). Double acceptance sampling based on truncated life tests in Rayleigh distribution, European Journal of Scientific Research, 17, 605-611.

[2] Aslam.M., and Kantam.R.R.L.(2008). Economic reliability acceptance sampling plan based on truncated life tests in Birnbaum-Saunders distribution, Pakistan Journal of Statistics, 24, $269-276$.

[3] Aslam.M., Jun.C.H., and Ahmad.M. (2009). A group sampling plan based on truncated life tests for gamma distribution, Pakistan Journal of Statistics, 25, 333-340.

[4] Aslam.M., and Jun.C.H. (2009a). A group sampling plan based on truncated life test having Weibull distribution, Journal of Applied Statistics, 36, 1021-1027.

[5] Aslam.M., Debasis Kundu., Jun.C.H., and Ahmad.M. (2011). Time truncated group acceptance sampling plans for generalized exponential distribution, Journal of Testing and Evaluation, 39(4), 968-976.

[6] Aslam.M.,Jun.C.H., Ahmad.M., and Rasool.M.(2011a). Group acceptance sampling plans for resubmitted lots under Burr-type XII distributions, Chinese Institute of Industrial Engineers, 28(8), 606-615.

[7] Baklizi.A. (2003). Acceptance sampling based on truncated life tests in the pareto distribution of the second kind, Advances and Applications in Statistics, 3, 33-48.

[8] Balakrishnan.,N.(1985). Order statistics from the half logistic distribution, Journal of Statistical Computation and Simulation, 20, 287-309.

[9] Balakrishnan.,N., Leiva.V., and Lopez.J. (2007). Acceptance sampling plans from truncated life tests based on generalized Birnbaum-saunders distribution, Communications in Statistics-Simulation and Computation, 36, 643-656. 
[10]Epstein.B. (1954) Truncated life tests in the exponential case, Annals of Mathematical Statistics, 25, 555-564.

[11]Fertig.F.W., and Mann.N.R. (1980). Life-test sampling plans for two-parameter Weibull populations, Technometrics, 22, 165-177.

[12]Govindaraju.K., and Ganesalingam.S.(1977). Sampling inspcetion for resubmitted lots, Communication in Statistics -Simulation and Computation, 26, 1163-1176.

[13]Gupta.S.S., and Groll.P.A. (1961). Gamma distribution in acceptance sampling based on life tests, Journal of the American Statistical Association, 56, 942-970.

[14]Gupta.S.S. (1962). Life test sampling plans for normal and lognormal distribution, Technometrics, 4, 151-175.

[15]Kantam.R.R.L., and Rosaiah.K.(1998). Half Logistic distribution in acceptance sampling based on life tests, IAPQR Transactions, 23(2), 117-125.

[16]Kantam.R.R.L., Rosaiah.K., and Rao.G.S.(2001). Acceptance sampling based on life tests, Log-logistic distribution, Journal of Applied Statistics, 28(1), 121-128.

[17]Lawless.J.F. (1982). Statistical models methods for lifetime data, John Wiley \& Sons, New York.

[18]Lio.Y.L., Tsai.T.R., and Wu.S.J. (2010). Acceptance sampling plans from truncated life tests based on Birnbaum-Saunders distribution for percentiles, Communications in StatisticsSimulation and Computation, 39, 119-136.

[19]Ramaswamy.A.R.S., and Anburajan.P. (2012). Group acceptance sampling plans using weighted binomial on truncated life tests for inverse Rayleigh and Log-logistic distributions, IOSR Journal of Mathematics, 2(3), 33-38.

[20]Rosaiah.K., and Kantam.R.R.L. (2005). Acceptance sampling plans based on inverse Rayleigh distribution, Economic Quality Control, 20(2), 277-286.

[21]Rosaiah.K., Kantam.R.R.L., and Rao.B.S. (2009). Reliability test plan for half logistic distribution, Calcutta Statistical Association, 61(241-244), 183-196.

[22]Rao.G.S., Ghitany.M.E., and Kantam.R.R.L.(2008). Acceptance sampling plans for Marshall-Olkin extended Lomax distribution, International Journal of Applied Mathematics, 21, 315-325. 
[23]Rao.G.S., Ghitany.M.E., and Kantam.R.R.L.(2009). Reliability test plans for Marshall-Olkin extended exponential distribution, Applied mathematical sciences , 3(55), 2745-2755.

[24]Rao.G.S. (2009). A group acceptance sampling plans for truncated life tests for MarshallOlkin extended Lomax distribution, Electronic Journal of Applied Statistical Analysis, 3(1), $18-28$.

[25]Rao.G.S., and Kantam.R.R.L.(2010). Acceptance sampling plans from truncated life tests based on log-logistic distribution for percentiles, Economic Quality Control, 25(2), 153167.

[26]Rao.G.S. (2011). A hybrid group acceptance sampling plans for lifetimes based on loglogistic distribution, Journal of Reliability and Statistical Studies, 4(1), 31-40.

[27]Rao.B.S., and Kantam.R.R.L.(2011). Software Reliability growth model based on half logistic distribution, Journal of Testing and Evaluation, 39(6), 1152-1157.

[28]Rao.B.S., and Kantam.R.R.L.(2011a). Significance test for half logistic distribution, Economic Quality Control, 26, 53-62.

[29]Rao.B.S., and Kantam.R.R.L.(2012). Mean and Range charts for skewed distributions -A comparison based on half logistic distribution, Pakistan Journal of Statistics, 28(4), 437444.

[30]Rao.B.S., and Kantam.R.R.L.(2012a). Extreme value charts and analysis of means based on half logistic distribution, International Journal of Quality and Reliability Management, 29(5), 501-511.

[31]Sultan.K.S.(2007). Order statistics from generalized exponential distribution and applications, Communications in Statistics-Theory and Methods, 36(7), 1409-1418.

[32]Tsai.T.R., and Wu.S.J.(2006). Acceptance sampling plans based on truncated life test for generalized Rayleigh distribution, Journal of Applied Statistics, 33, 595-600.

[33]Wu.C.J.,and Tsai.T.R.(2005). Acceptance sampling plans for Birnbaurn-Saunders distribution under truncated life tests, International Journal of Reliability, Quality and Safety Engineering, 12, 507-519.

Received September 11, 2013; accepted May 6, 2014. 
G.Srinivasa Rao

Department of Statistics

The University of Dodoma

Dodoma, P.O.Box: 259, Tanzania.

gaddesrao@yahoo.com

B.Srinivasa Rao

Department of Mathematics \& Humanities

R.V.R \& J.C College of Engineering

Chowdavaram, Guntur-522 019, Andhra Pradesh, India.

boyapatisrinu@yahoo.com 
